\title{
SCMR: Static Clustering based Multi-hop Routing in Wireless Sensor Network
}

\author{
Hamta Sedghani ${ }^{1}$ and Mina Zolfy Lighvan ${ }^{2}$ \\ ${ }^{1,2}$ Faculty of electrical and computer engineering, University of Tabriz, Tabriz, Iran \\ E-mail: ${ }^{1}$ h.sedghani91@ms.tabrizu.ac.ir, ${ }^{2}$ mzolfy@tabrizu.ac.ir
}

\begin{abstract}
The most challengeable issue in wireless sensor networks is the limited energy of their nodes that are distributed in a field for collecting information from the environment. Thus, energy efficiency and lifetime of these networks consider one of important and controversial issues in this field. In this paper, a new energy effective routing algorithm is presented which is based on static clustering and multi-hop transmission. The SCMR (Static Clustering Based Multi-hop Routing) Algorithm is verified with MATLAB simulator. Simulation results show that the new method compared to previous methods such as LEACH, could balances the energy consumption, thus increase the stable period of network.
\end{abstract}

Keywords: Wireless sensor networks; energy efficiency; Static Clustering; Multi-hop Routing; MATLAB.

\section{INTRODUCTION}

Wireless sensor networks (WSN) are new generation of networks which typically consist of many inexpensive nodes and these nodes can be connected wirelessly. The main purpose of these networks is collection of information data about the environment. Overall function of these networks is that the nodes collect the necessary information and then send to the receiver. Dissemination of information in these networks is largely similar to ad-hoc networks. The major difference of ad-hoc networks and WSNs is their limited energy resources and low processing capability. Therefore the diffusion and transmission of information in these networks is very important because incorrect routing will result in imbalance energy consumption and it will cause the energy level of nodes reduce quickly and the nodes die soon. So if we use the methods which increase nodes lifetime, the network lifetime will increased [1].

Each sensor node of WSN is consist of four blocks namely, sensing unit, processing unit, communication unit and power unit. The sensing unit measures a physical condition like pressure and temperature in the environment. The processing unit collects and processes signals obtained from sensors. The wireless communication unit is responsible for transferring signals. The power unit provides the required energy in order to carry out the mentioned tasks [2].

The sensors are usually distributed in areas that are not accessible by humans, such as forests or in war zones. So the sensors are not usually rechargeable. The sensors transmit their collected data to a BS (Base Station). Routing algorithm for the transmission of sensed data to the BS may play a significant role in reducing the energy consumption of sensors. In aspect of routing protocol, there are two different solutions from existing works, the first of them is flat routing and the second is hierarchical routing. In flat routing, each sensor node has the same role and sends its data to sink node directly which always results in faster energy consumption and excessive data redundancy. In hierarchical routing, the field is divided into several clusters, in accordance with the distance between the nodes and the hop count. For collecting the data from various sensor nodes, there is a requirement of collection point called cluster head. Therefore, each cluster consists of some sensor nodes and a cluster head [3]. Clustering is one of the most popular ways for routing in this type of network. Many routing protocol are based on this method, for example LEACH [4], DEEC [5], TEEN [6] and SEP [7] are some of them. In clustering algorithms, a cluster head is typically selected for each cluster, which is responsible for 
receiving information from other nodes to collect and combine information and finally send it to the destination. Another routing method is data transmission from source to destination as a chain which was introduced and named "PEGASIS". PEGASIS uses a greedy algorithm for transmit data to the BS [8]. In this paper, we propose a new method SCMR (Static Clustering based Multi-hop Routing), that is a hierarchical static clustering based method. SCMR omits the overhead of dynamic clustering and reduce the energy consumption and as a result prolongs the network lifetime. It uses static clustering and multi-hop routing. In this method, the clusters can be fixed, but cluster heads are variable in each round.

Then, in Section 2, we'll see the related work done in the past. The proposed method will be described in Section 3. In Section 4 we will show the result of simulation. And then, in Section 5, conclusions are done.

\section{RELATED WORK}

So far, several routing algorithms have been presented for wireless sensor networks. One of the clustering based algorithms is Low Energy Adaptive Clustering Hierarchy (LEACH). It is an adaptive and self-organized clustering protocol proposed by Heinzelman [4, 9, 10]. The operation of LEACH is composed of some rounds where each round begins with a setup phase followed by a steady-state phase.

The clusters are organized in setup phase, and then in the steady state phase the data is transferred from nodes to the $\mathrm{CH}$ and then to the BS.

In this algorithm, at the beginning of round each node makes the decision of being the cluster head or not. This decision is made based on the random number generated by the node. If the value of T(n) function was smaller than the generated random number, the node will be selected as the cluster head. Otherwise, the node will be a normal node. $T(n)$ is calculated as equation (1) [4].

$$
\mathbf{T}(\mathbf{n})= \begin{cases}\frac{P}{1-P *(r \bmod 1 / P)} & \text { if } n \in G \\ 0 & \text { otherwise }\end{cases}
$$

$\mathrm{P}$ is the percentage of total nodes that will be selected as cluster head. $r$ is the number of current round. $\mathrm{G}$ is the set of the nodes that have not been cluster head in the first $1 / \mathrm{P}$ rounds. When the clusters were formed, the nodes begin to transmit data to their cluster heads. Cluster heads also sends received data to the $\mathrm{BS}$.
Another well-known algorithm is PEGASIS [11]. The main idea of this method is that each node receives data from its nearest neighbor and sends it to the next nearest neighboring. In this way, data transmit between the nodes as a chain. In every round, one node is randomly selected as the leader. The leader receives information from the neighboring nodes, collects and combines it, then sends it to the BS.

For example, in Figure 1, C2 is chosen as the leader node and receive data from $\mathrm{C} 1$ and $\mathrm{C} 0$ and then sends it to the BS. So each node will be selected as the leader one time in each $\mathrm{n}$ rounds [11, $12]$.

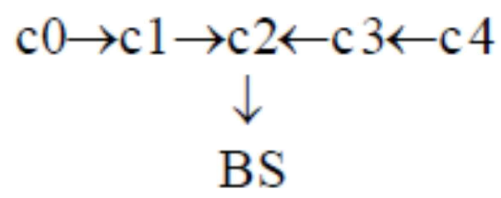

Fig. 1. Chain in PEGASIS

Hierarchical clustering algorithms increased lifetime of WSN considerably [13].The paper focused on heterogeneity of nodes regarding their energy. It is assumed that the sensor nodes were equipped with small amount of energy and the nodes were not mobile. It was also given that the heterogeneous networks contained two types of nodes namely, type- 1 node and type- 0 node, where type-1 node was having more battery power than type- 0 node. Cluster formation and cluster head selection was based on weighted election probabilities of each node.

In [14] an energy aware routing protocol was proposed to perform admission control, appointment of bandwidth requirements and the evaluation of sensor's residual energy. By adopting selective forwarding method in accordance with sensor location, the delay of carried flows was optimized. The major limitation regarding this work is that the work completed with static sensor nodes without the consideration of node's mobility.

A coverage based energy efficient algorithm was proposed in [15]. It was explained in the paper that multi-hop short range communication among the sensor nodes were more energy efficient than single-hop long range communication comparatively. Moreover, the paper was involved in making uniform distribution of CHs using nonoverlapped cluster areas. The main purpose was to achieve higher packet reception rate irrespective to network longevity.

Another work in [16] proposed MG-LEACH (Multi Group Based LEACH) that also focused the aforementioned criteria in a different dimension 
such that same redundant nodes were located in the same area.

A review on energy efficient protocols for WSN was given in [17].

\section{PROPOSED METHOD}

As explained before the SCMR method is a static clustering based multi-hop routing method presented for WSNs.

\subsection{SCMR METHOD}

In this method, the field is divided into three nested concentric circles where the sink node is in the center of these circles. Each area of a circle minus its inner circle forms ring shape area which shows a group. With such a partitioning three main groups are formed. The radiuses of the circles are determined in a way that the area of the closer group to the sink becomes smaller than the area of the farther once. With these criteria the inner groups will hold fewer nodes than the outer once. Each main group is divided into four sub-groups. In this way, each circle is divided into four equal parts by two crossover lines which are numbered from one to four. Each partition obtained from the last division is considered as a cluster. Thus, the clustering type is static and clusters will be formed before the network formation and will not be changed until the end of its lifetime. Fig3 shows the field partitioning required for SCMR method.

This model uses multi-hop data transmission. Each cluster head collects data from the normal nodes of the same cluster and sends it to the next cluster head. Here next cluster means the cluster in the closer inner group with the same sub-group number. This work continues until the node in the innermost main group receives information and sends it to the sink. To select the cluster heads in each round, the node with the greatest amount of energy will be selected as the cluster heads.

Dividing environment into two types of main groups and sub groups has two advantages:

1) As mentioned before, the main groups closer to the sink are spatially smaller than the farther groups [18]. Therefore the clusters formed near the sink are smaller than the farther clusters. Thus, cluster heads closer to the BS can spend more energy to transmit data between clusters.

2) The advantage of dividing each main group into four sub-groups is making the distribution of cluster heads more monotonously. It causes the cluster heads not to be concentrated in an area, and by scattering the cluster heads, the data transmission distance will be shorten.

For example, in figure2 (a), suppose that you have a circle shaped field which should contain four cluster heads, if cluster head selection being done in a random manner, it is possible to have some close together cluster heads. In this way the maximum distance between some normal nodes and their corresponding cluster heads may be as large as the circle diameter that causes the high power consumption. But with dividing the circle into four equal parts, such as figure 2 (b), and assign a cluster head in the each distinct part, the distance will be shorter which implies less energy consumption.

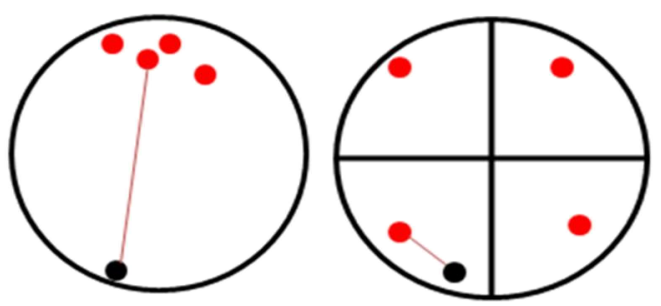

Fig. 2. (a) Choosing the cluster heads randomly in total of circle. (b) Choosing the cluster heads in each of the four parts.

\section{$3.2 A N E X A M P L E$}

The proposed method is illustrated as following: Figure 3, shows an area in which nodes A, B and C are selected as the cluster heads in Sub group number 2 in three main groups 1,2 and 3 respectively.

Node $\mathrm{C}$, located in the main group3 and subgroup2, collects data from normal nodes of its cluster and send it to node B, which located in the main group2 and sub-group2. Node B collects data from normal nodes of its cluster and fuses data received from node $\mathrm{C}$ with its own and then sends to node $\mathrm{A}$, which located in the main group1 and sub-group2. Node A collects data from ordinary nodes of its cluster and fuses it with node B's data and then sends all to the sink.

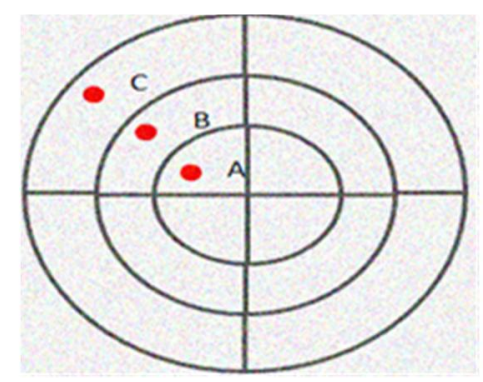

Fig. 3. SCMR's model 


\subsection{FIRST-ORDER WIRELESS TRANSMISSION MODEL}

First-order wireless communication model has been used for data transmission in this paper, which is shown in Figure 4.

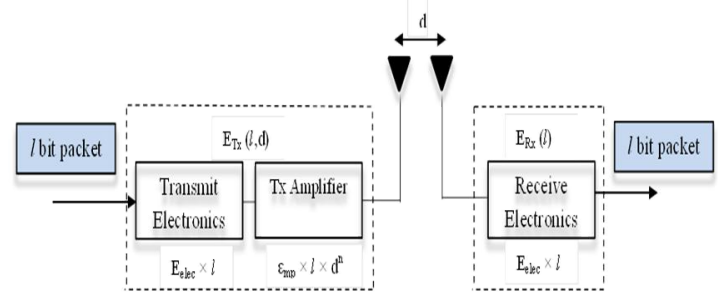

Fig. 4. The wireless communication model.

The total energy consumed in the model showed in Figure 5 is calculated by formula (2) and (3) [19]:

$E_{T x}(\mathrm{~L}, \mathrm{~d})= \begin{cases}L E_{\text {elec }}+L \varepsilon_{f s} d^{2}, & \mathrm{~d} \leq d_{0} \\ L E_{\text {elec }}+L \varepsilon_{m p} d^{4}, & \mathrm{~d}>d_{0}\end{cases}$

$E_{R x}(\mathrm{~L})=L E_{\text {elec }}$

Where $E_{\text {elec }}$ represents the energy consumed to send or receive one bit message, $\varepsilon_{f s}$ is the amplification coefficient of free-space signal, $\varepsilon_{m p}$ is the multi-path fading signal amplification coefficient, their value depends on the circuit amplifier model; $d$ represents the distance between sender and receiver; $\mathrm{L}$ is the bit amount of sending information.

$d_{0}$ is calculated by formula (4) [19]:

$$
d_{0}=\sqrt{\frac{\varepsilon_{f s}}{\varepsilon_{m p}}}
$$

\section{SIMULATION AND ANALYSES}

Matlab7.8.0 [20] has been used as simulation platform to evaluate the performance of the new algorithm SMCR and compare it with LEACH protocol.

\subsection{Simulation Parameters}

Simulation scenarios in this paper are:

1. Sensor nodes are distributed in a square region randomly.

2. Sensor nodes are homogeneous and each node has a unique ID number throughout the network and nodes' energy is limited. The node's site is fixed after deployed.
3. The base station is in the center of circles with fixed location.

4. Nodes communicate with BS via singlehop or multi-hop

In this simulation, 200 nodes randomly within the square area of the $100 \mathrm{~m} * 100 \mathrm{~m}$, the base station is located in the center of the circles, the base station coordinates is $(50,50)$. We consider the radius of inner circle and second circle, 25 and 45 respectively. Figure 5 shows the simulation environment for this model and node distribution in the field.

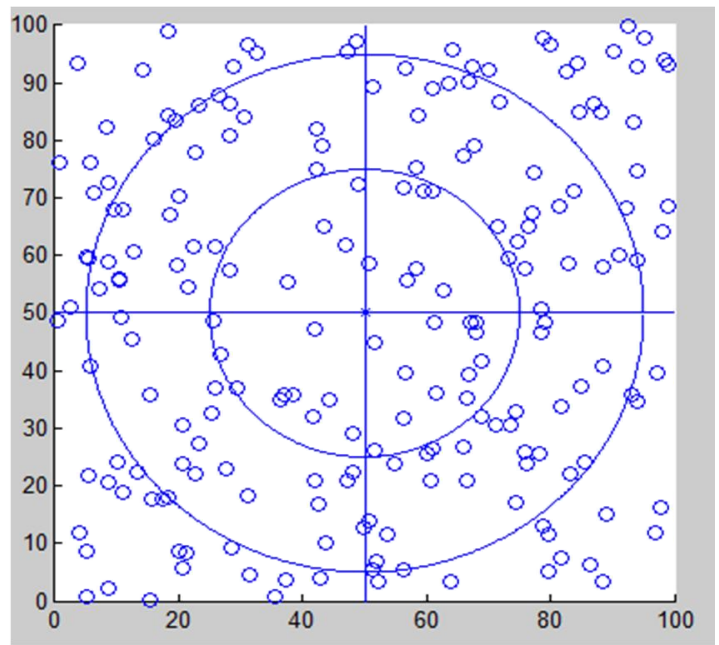

Fig. 5. Simulation environment in matlab.

Specific parameters are shown in table 1.

Table. 1. SIMULATION ENVIRONMENT PARAMETERS

\begin{tabular}{|l|l|}
\hline Parameters & Parameters \\
\hline area & Packet size \\
$100 * 100$ & $4000 \mathrm{bits}$ \\
\hline Nodes number & Eelec \\
200 & $50 \mathrm{~nJ} / \mathrm{bit}$ \\
\hline Initial energy & Efs \\
$0.5 \mathrm{~J}$ & $10 \mathrm{pJ} / \mathrm{bit} / \mathrm{m} 2$ \\
\hline $\mathrm{CH}$ proportion & Emp \\
$\mathrm{p}=7 \%$ & $0.0013 \mathrm{pJ} / \mathrm{bit} / \mathrm{m} 4$ \\
\hline BS location & EDA \\
$(50,50)$ & $5 \mathrm{~nJ} / \mathrm{bit}$ \\
\hline
\end{tabular}

\subsection{Analysis of simulation results}

In WSN, the network life is divided into stable and unstable period. Stable period usually means 
the time from the beginning of the simulation to the time when the first node dies, the unstable period refers to the time from the death of first node to the end of simulation.

When nodes start to die, the network operation may become unstable and data transfer may become unreliable. Therefore, the longer stable period causes the higher network performance.

The proposed method increases the stable period of the network compared to the LEATCH method which uses a dynamic clustering protocol.

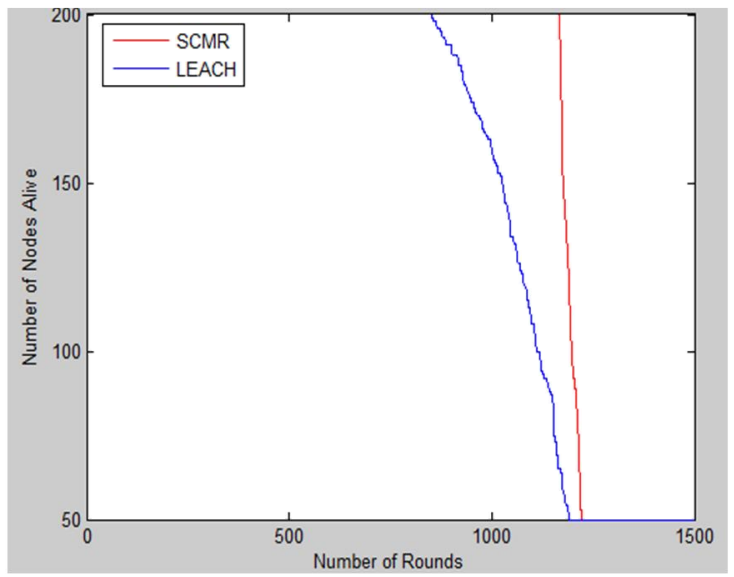

Fig. 6. The network lifetime of the first 150 nodes.

Figure 6 shows network life time until the death 150 nodes. First node dies in the round 838 in LEACH protocol where in the new proposed method the first node dies in the round 1165 . Therefore, the proposed method increases the network stable period to about 300 rounds. The percentage of stable period in whole lifecycle of the network in LEACH Protocol is 55\% where in our SCMR protocol is $86 \%$. The stable period percentage in SCMR algorithm has $31 \%$ improvement. This indicates that the performance of improved protocol is much better than the LEACH Protocol.

Figure 7 shows the network lifetime for total nodes of network.

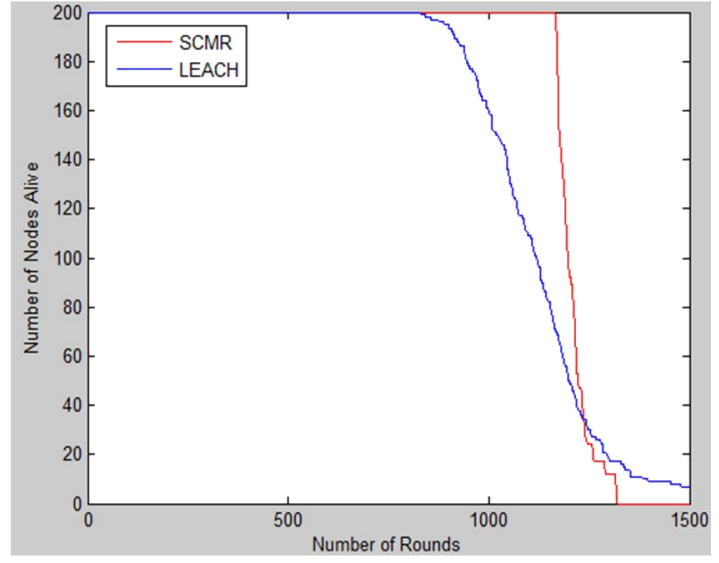

Fig. 7. The network lifetime of total nodes.

Figure 8 shows the energy consumption diagram which proves that the proposed method consume lower energy compared to LEACH protocol.

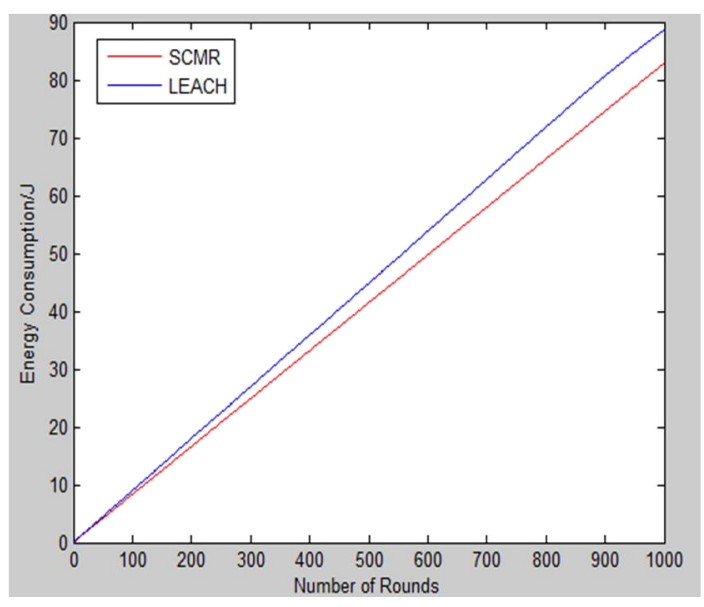

Fig. 8. The total energy consumption.

\section{CONCLUSION}

In this paper, we introduce a novel Static Clustering based Multi-hop Routing method for wireless sensor networks. In the proposed SCMR method with partitioning the field into three main 
groups and again partitioning each main groups to four sub-groups a new scheme for cluster head assigning has been defined. Then a new method using multi-hop routing and static clustering is presented. Matlab simulation results of these methods proves that SCMR method in compared to the algorithms with dynamic clustering such as LEACH has lower energy consumption and longer network lifetime.

\section{REFERENCES}

[1] SOHRABY, K., D. MINOLI, and T. ZNATI, WIRELESS SENSOR NETWORKS Technology, Protocols, and Applications. 2007.

[2] Neha, J., "Energy Efficient And Cluster Based Routing Protocol For Wireless Sensor Network:A Review,". International Journal of Advance Technology \& Engineering Research, 2011. 1(1).

[3] Chen, Y. and Y. Chen. "An Energy Efficient Clustering Algorithm Based on Residual Energy and Concentration Degree in Wireless Sensor Networks,". in In the Proceedings of the Second Symposium International Computer Science and Computational Technology,. 2009.

[4] Heinzelman, W., A. Chandrakasan, and H. Balakrishnan. Energy Efficient Communication Protocol for Wireless Microsensor Netwroks (LEACH). in Proceedings of 33rd Hawaii international conference systems science. 2004.

[5] Qing, L., Q. Zhu, and etc, "Design of a distributed energy-efficient clustering algorithm for heterogeneous wireless sensor networks". Computer Communications, August, 2006. 29(12): p. 2230-2237.

[6] Manjeshwar, A. and D.P. Agarwal, "TEEN: a routing protocol for enhanced efficiency in wireless sensor networks", in 1st International Workshop on Parallel and Distributed Computing Issues in Wireless Networks and Mobile Computing. April 2001.

[7] Smaragdakis, G., I. Matta, and A. Bestavros, "SEP: A stable election protocol for clustered heterogeneous wireless sensor networks". Boston University Computer Science Department, 2004.

[8] Akyildiz, I.F., et al., "Wireless Sensor Networks: A Survey". Computer Networks, 2002: p. 393-442.

[9] Ozdemir, S. and Y. Xiao, "Secure data aggregation in wireless sensor networks: A comprehensive overview,". Computer Networks,, August 2009. 53: p. 2022-2037.
[10]Lee, H.S., K.T. Kim, and H.Y. Youn, "A New Cluster Head Selection Scheme for Long Lifetime of Wireless Sensor Networks,". ICCSA, 2006. 3983.

[11] Lindsey, S. and C.S. Raghavendra. PEGASIS: Power-efficient gathering in sensor information systems. in Proceedings of the IEEE Aerospace Conference. 2002. Montana: Big Sky.

[12]FU1, C., et al., " An Energy Balanced Algorithm of LEACH Protocol in WSN". International Journal of Computer Science, January 2013.

[13] Kumar, D., T.C. Aseri, and R.B. Patel, "EEHC: Energy efficient heterogeneous clustered scheme for wireless sensor networks,". Journal of Computer Communications, 2009. 32: p. 662-667.

[14]Koulali, M.A., et al. "A QoS-Geographic and Energy Aware Routing Protocol for Wireless Sensor Networks,". in In the Proceedings of International Symposium on I/V Communications and Mobile Network (ISVC). 2007.

[15] Thakkar, A. and K. Kotecha, "CVLEACH: Coverage based energy efficient LEACH,". International Journal of Computer Science and Network, 2012. 1(3).

[16]Haneef, H., Z. Wenxun, and Z. Deng. "MGLEACH: Multi Group Based LEACH an Energy Efficient Routing Algorithm for Wireless Sensor Network,". in In the Proceedings of 14th International Conference on Advanced Communication Technology. 2012.

[17]B.Baranidharan and B.Shanthi., "A Survey on Energy Efficient Protocols for Wireless Sensor Networks,". International Journal of Computer Applications,, 2010. 11(10): p. 35- 40.

[18] Bai, F., H. Mou, and J. Sun, ” Power-Efficient Zoning Clustering Algorithm for Wireless Sensor Networks", in Information Engineering and Computer Science. 2009: Wuhan.

[19] Smaragdakis, G., I. Matta, and A. Bestavros. "A Stable Election Protocol for Clustered Heterogeneous Wireless Sensor Networks". in Proceedings of the 2nd International Workshop on SANPA 2004. 2004:1-11. Massachusetts, U.S.

[20] Higham, D.J. and N.J. Higham, MATLAB Guide 2005. 